\title{
Drawing 3-Polytopes with Good Vertex Resolution
}

\author{
André Schulz \\ MIT, Computer Science and Artificial Intelligence Laboratory, Cambridge, USA \\ schulz@csail.mit.edu
}

\begin{abstract}
We study the problem how to obtain a small drawing of a 3-polytope with Euclidean distance between any two points at least 1. The problem can be reduced to a one-dimensional problem, since it is sufficient to guarantee distinct integer $x$-coordinates. We develop an algorithm that yields an embedding with the desired property such that the polytope is contained in a $2(n-2) \times 1 \times 1$ box. The constructed embedding can be scaled to a grid embedding whose $x$-coordinates are contained in $[0,2(n-2)]$. Furthermore, the point set of the embedding has a small spread, which differs from the best possible spread only by a multiplicative constant.
\end{abstract}

\section{Introduction}

Let $G$ be a 3 -connected planar graph with $n$ vertices $v_{1}, \ldots, v_{n}$ and edge set $E$. Due to Steinitz' seminal theorem [16] we know that $G$ admits a realization as 3-polytope, and every edge graph of a 3-polytope is planar and 3-connected. The question arises how one can obtain a "nice" realization of a 3-polytope when its graph is given. One particular property which is often desired from an aesthetically point of view is that the vertices of the embedding should be evenly distributed. If two vertices lie too close together they are hard to distinguish. Such an embedding may appear as bad "illustration" for the human eye. Of course we can always scale a 3-polytope to increase all of its pointwise distances, but clearly this is not the right solution to create a good drawing. Therefore, we restrict ourselves to an embedding whose vertices have, pairwise, an Euclidean distance of at least 1 . We say that in this case the embedding/drawing is under the vertex resolution rule. See [1] for a short discussion on resolution rules. Notice, that the resolution rule depends on a particular distance measure. Throughout the paper we use the Euclidean distance, but our results can be easily modified for the $L_{1}$ distance.

In 2d, drawings of planar graphs can be realized on an $O(n) \times O(n)$ grid [145]. Since the grid is small, these grid embeddings give a good vertex resolution for free. The situation for 3-polytopes is different. The best known algorithm uses a grid of size $O\left(2^{7.55 n}\right)$ [12]. Thus, the induced resolution might be bad for this grid embedding.

\footnotetext{
* Supported by the German Research Foundation (DFG) under grant SCHU 2458/1-1.
} 
Let us briefly discuss some approaches how to realize $G$ as 3-polytope. Steinitz' original proof is based on a transformation of $G$ to the graph of the tetrahedron. The transformation consists of a sequence of local modifications which preserve the realizability of $G$ as 3-polytope. However, the proof does not include a direct method how to construct the 3 -polytope given by $G$. The KoebeAndreev-Thurston Theorem on circle packings gives a more constructive proof of Steinitz' Theorem (see for example Schramm [15). This approach relies on non-linear methods which makes many geometric features of the constructed 3-polytope intractable. A third approach uses liftings of planar graphs with equilibrium stresses (known as the Maxwell-Cremona correspondence [18]). This powerful method is used in a series of embedding algorithms [16 81312. A completely different approach is due to Das and Goodrich 4. It uses an incremental technique which only needs $O(n)$ arithmetic operations for embedding $G$ as 3-polytope, but works only for triangulated planar graphs.

Results. We show how to obtain an embedding of $G$ as 3-polytope inside a $2(n-2) \times 1 \times 1$ box under the vertex resolution rule. It is even possible to make the box arbitrarily flat such that its volume gets arbitrarily small. But for aesthetic reasons we leave the side lengths at least 1 . Our algorithm is based on the Maxwell-Cremona approach and extends the ideas of [12. In contrast to the construction of 12 we can handle more complicated interior edge weights (stresses). Our algorithm creates an embedding with two more interesting properties: (1) it can be scaled to a grid embedding whose $x$-coordinates are in $[0,2(n-2)]$, and (2) the point set of the embedding has a good ratio between its largest and shortest pointwise distance. We show that this number differs only by a constant from the best possible ratio. Due to space constraints we omit the proofs for Theorem 3 and 4 . The proofs can be found in the full version of the paper, which is available on the website of the author. The full version includes also an example of the algorithm.

Related work. In [1] Chrobak, Goodrich, and Tamassia introduced an algorithm that embeds a 3-polytope with good vertex resolution. However, their result was only published as preliminary version, without giving all details. Moreover, their algorithm is only applicable for polytopes that contain a triangular face. We will reuse some of their ideas for our algorithm, but especially the complicated setting where the polytope does not have a triangular face requires completely new techniques.

\section{Preliminaries: Maxwell and Tutte}

Since $G$ is 3-connected and planar, the facial structure of $G$ is uniquely determined [19. Let us pick an arbitrary face $f_{b}$, which we call the boundary face. We assume further that the vertices are ordered such that $v_{1}, v_{2}, \ldots, v_{k}$ are the vertices of $f_{b}$ in cyclic order. An edge (vertex) is called boundary edge (vertex) if it lies on $f_{b}$, otherwise interior edge (vertex). In this paper every embedding is considered as straight-line embedding. A $2 \mathrm{~d}$ embedding of $G$ is described by 
giving every vertex $v_{i}$ a coordinate $\mathbf{p}_{i}=\left(x_{i}, y_{i}\right)^{T}$. We denote the $2 \mathrm{~d}$ embedding with $G(\mathbf{p})$ and consider only embeddings that realize $f_{b}$ as convex outer face.

The combination of the Maxwell-Cremona correspondence and barycentric embeddings provides an elegant technique for embedding 3-polytopes. Let us first define the common concept of both methods.

Definition 1 (Stress, Equilibrium). An assignment $\omega: E \rightarrow \mathbb{R}$ of scalars to the edges of $G$ (with $\left.\omega(i, j)=: \omega_{i j}=\omega_{j i}\right)$ is called a stress. Let $G(\mathbf{p})$ be a $2 d$ embedding of $G$. A point $\mathbf{p}_{i}$ is in equilibrium, iff

$$
\sum_{j:(i, j) \in E} \omega_{i j}\left(\boldsymbol{p}_{i}-\boldsymbol{p}_{j}\right)=\mathbf{0} .
$$

The embedding of $G$ is in equilibrium, iff all of its points are in equilibrium.

If $G(\mathbf{p})$ is in equilibrium according to $\omega$, then $\omega$ is called equilibrium stress for $G(\mathbf{p})$. From special interest are stresses that are positive on every interior edge of $G$. These stresses are called positive stresses.

Suppose we fixed an embedding $G(\mathbf{p})$ in the plane. Let $h_{i}: V \rightarrow \mathbb{R}$ be a height assignment for the vertices of $G$. The function $h$ defines a $3 \mathrm{~d}$ embedding of $G$ by giving every vertex $\mathbf{p}_{i}$ the additional $z$-coordinate $z_{i}=h\left(\mathbf{p}_{i}\right)$. If in the $3 \mathrm{~d}$ embedding every face of $G$ lies on a plane the height assignment $h$ is called lifting. The so-called Maxwell-Cremona correspondence describes the following.

Theorem 1 (Maxwell [11], Whiteley). Let $G$ be a 3-connected planar graph with $2 d$ embedding $G(\mathbf{p})$ and a designated face $\hat{f}$. There exists a correspondence between

A.) equilibrium stresses $\omega$ on $G(\mathbf{p})$,

B.) liftings of $G(\mathbf{p})$ in $\mathbb{R}^{3}$, where face $\hat{f}$ lies in the $x y$-plane.

If the stress is positive, the lifting refers to a convex 3-polytope.

The complete proof of the Maxwell-Cremona correspondence is due to Whiteley [18] (see also [3]). A more constructive proof with detailed rules how o compute the lifting can be found in Richter-Gebert's book 13 .

To apply the Maxwell-Cremona correspondence we need a $2 \mathrm{~d}$ embedding of $G$ with equilibrium stress. Let $\omega$ be an arbitrary stress that is zero on the boundary edges and positive on the interior edges. We obtain from the stress $\omega$ its Laplace matrix $L=\left\{l_{i j}\right\}$, which is defined by its entries as

$$
l_{i j}:=\left\{\begin{array}{cl}
-\omega_{i j} & \text { if }(i, j) \in E \text { and } i \neq j \\
\sum_{j} \omega_{i j} & \text { if } i=j, \\
0 & \text { otherwise. }
\end{array}\right.
$$

An embedding $G(\mathbf{p})$ is described by the vectors $\mathbf{x}=\left(x_{1}, \ldots, x_{n}\right)^{T}$ and $\mathbf{y}=$ $\left(y_{1}, \ldots, y_{n}\right)^{T}$. Let $B:=\{1, \ldots, k\}$ and $I:=\{k+1, \ldots, n\}$ be the index sets of the boundary, respectively interior vertices. We subdivide $\mathbf{x}, \mathbf{y}$, and $L$ by $B$ and $I$ and obtain $\mathbf{x}_{B}, \mathbf{x}_{I}, \mathbf{y}_{B}, \mathbf{y}_{I}, L_{B B}, L_{I I}, L_{B I}$ and $L_{I B}$. The equilibrium condition 
(11) for the interior vertices can be phrased as $L_{I B} \mathbf{x}_{B}+L_{I I} \mathbf{x}_{I}=\mathbf{0}$. The same holds for the $y$-coordinates. This implies that we can express the vectors $\mathbf{x}_{I}$ and $\mathbf{y}_{I}$ as linear functions in terms of $\mathbf{x}_{B}$ and $\mathbf{y}_{B}$, namely by

$$
\mathbf{x}_{I}=-L_{I I}^{-1} L_{I B} \mathbf{x}_{B}, \quad \mathbf{y}_{I}=-L_{I I}^{-1} L_{I B} \mathbf{y}_{B} .
$$

The matrix $L_{I I}$ is singular and hence the vectors $\mathbf{x}_{I}$ and $\mathbf{y}_{I}$ are properly and uniquely defined (see [137]). The embedding is known as (weighted) barycentric embedding. For every positive stress the coordinates give a strictly convex straight-line embedding (see for example [7]). A weighted barycentric embedding has the following nice interpretation: The interior edges of the graph can be considered as springs with spring constants $\omega_{i j}$. The barycentric embedding models the equilibrium state of the system of springs when the boundary vertices are anchored at their fixed positions.

\section{Extending an Equilibrium Stress to the Boundary}

The interior vertices computed by (2) are in equilibrium by construction. However, the stressed edges of the boundary vertices wont sum up to zero but to

$$
\forall i \in B \quad \sum_{j:(i, j) \in E} \omega_{i j}\left(\mathbf{p}_{i}-\mathbf{p}_{j}\right)=: \mathbf{F}_{i} .
$$

To apply the Maxwell-Cremona correspondence we have to define the (yet unassigned) stresses on the boundary edges such that they cancel the vectors $\mathbf{F}_{i}$. If the outer face is a triangle this is always possible by solving a linear system with three unknowns [8]. But in general this is only possible for special locations of the boundary face. The problem how to position $f_{b}$ is challenging, because changing the location of the boundary face will also change the vectors $\mathbf{F}_{i}$. We use the approach of Ribó, Rote, and Schulz [12] to express this dependence and obtain a formalism that helps us to extend the equilibrium stress to the boundary.

Lemma 1 (Substitution Lemma [12]). There are positive weights $\tilde{\omega}_{i j}=\tilde{\omega}_{j i}$, for $i, j \in B$, independent of location of the boundary face such that

$$
\forall i \in B \quad \mathbf{F}_{i}=\sum_{j \in B: j \neq i} \tilde{\omega}_{i j}\left(\mathbf{p}_{i}-\mathbf{p}_{j}\right) .
$$

The weights $\tilde{\omega}_{i j}$ are the off-diagonal entries of $-L_{B B}+L_{B I} L_{I I}^{-1} L_{I B}$, which is the Schur Complement of $L_{I I}$ in $L$ multiplied with -1 .

With help of the substitution stresses we can simplify the problem how to locate the boundary face. A feasible position can be found by solving the non-linear system consisting of the $2 k$ equations of (4) plus the $2 k$ equations

$$
\forall i \in B: \omega_{i, \operatorname{suc}(i)}\left(\mathbf{p}_{i}-\mathbf{p}_{\text {suc }(i)}\right)+\omega_{i, \operatorname{pre}(i)}\left(\mathbf{p}_{i}-\mathbf{p}_{\text {pre }(i)}\right)=-\mathbf{F}_{i},
$$

where $\operatorname{suc}(i)$ denotes the successor of $v_{i}$ and pre $(i)$ denotes the predecessor of $v_{i}$ on $f_{b}$ in cyclic order. Since the equations are dependent the system is underconstrained. We fix some boundary coordinates to obtain a unique solution. 


\section{Constructing and Controlling an $x$-Equilibrium Stress}

Let $x_{1}, \ldots, x_{n}$ be given as $x$-coordinates of $G(\mathbf{p})$. We are interested in a positive equilibrium stress that will give these $x$-coordinates in the barycentric embedding. In particular, we are looking for a positive stress $\omega$ such that

$$
\forall i \in I \sum_{j:(i, j) \in E} \omega_{i j}\left(x_{i}-x_{j}\right)=0 .
$$

We call a positive stress that fulfills condition (6) a (positive) $x$-equilibrium stress. Since we consider in this paper only positive $x$-equilibrium stresses we omit the term "positive" in the following. As pointed out in [1], an $x$-monotone stress exists when every interior edge lies on some $x$-monotone path, whose endpoints are boundary vertices. Let us assume that we selected the $x_{i}$ values such that the latter holds. Furthermore, we pick for every edge $e$ some $x$-monotone path $P_{e}$.

We follow the approach of 1 to construct an $x$-monotone stress. The construction is based on assigning a cost $c_{\{i, j\}}>0$ to every interior edge $(i, j)$ of $G$. If the costs guarantee

$$
\forall i \in I \sum_{j:(i, j) \in E: x_{i}<x_{j}} c_{\{i, j\}}=\sum_{(i, k) \in E: x_{i}>x_{k}} c_{\{i, k\}},
$$

we can define an $x$-equilibrium stress by

$$
\omega_{i j}=\frac{c_{\{i, j\}}}{\left|x_{i}-x_{j}\right|} .
$$

We start with $c_{\{i, j\}} \equiv 0$ and increase the costs successively. Let $e=(i, j)$ be an edge with $c_{\{i, j\}}=0$. We increase the costs of the edges of $P_{e}$ by 1 . In (77) both sides of the equation increase by 1 if $v_{i}$ lies on $P_{e}$, otherwise nothing changes. Hence, (7) still holds. We repeat this procedure until every interior edge is assigned with a positive cost. The total cost for an edge is an integer smaller than $3 n-3$.

We show now how to modify an $x$-monotone stress to obtain helpful properties for the substitution stresses induced by $\omega$. Our goal is to get a substitution stress that is maximal on an edge we picked. More precisely, let $v_{s}$ and $v_{t}$ be two nonincident vertices on the boundary of $G$ and let $\alpha>1$ be a constant that we fix later. The stress $\omega$ should guarantee that for all other pairs of boundary vertices $v_{i}, v_{j}$ we have $\tilde{\omega}_{s t}>\alpha \tilde{\omega}_{i j}$, unless $i j=s t$. The idea how to achieve this is the following: We take an $x$-monotone path $P_{s t}$ from $v_{s}$ to $v_{t}$. Then we take a suitable (large) number $K$ and add $K$ to the costs of every edge which is on $P_{s t}$. The stress induced by the increased costs is still an $x$-equilibrium stress for our choice of $x$-coordinates. But if we think of the stresses as spring constants we have increased the force that pushes $v_{s}$ and $v_{t}$ away from each other. Our hope is that this will reflect in the substitution stresses and makes $\tilde{\omega}_{s t}$ the dominant stress.

First, let us bound all substitution stresses form above and then prove a lower bound for the stress $\tilde{\omega}_{s t}$. 
Lemma 2. Let $L=\left\{l_{i j}\right\}$ be the Laplace matrix derived from a positive stress $\omega$, and $\tilde{\omega}$ the corresponding substitution stress. For any $i, j \in B$ we have

$$
\tilde{\omega}_{i j}<\min \left\{l_{i i}, l_{j j}\right\} \text {. }
$$

Proof. Since $\mathbf{u}^{T} L \mathbf{u}=\sum_{(i, j) \in E} \omega_{i j}\left(u_{i}-u_{j}\right)^{2}$, which is non-negative for any vector $\mathbf{u}$, the Laplace matrix is positive semidefinite. Let $\tilde{L}=\left\{\tilde{l}_{i j}\right\}=L_{B B}-$ $L_{B I} L_{I I}^{-1} L_{I B}$ denote the Schur complement of $L_{I I}$ in $L$. Due to [20, page 175] we know that $L_{I I}-\tilde{L}$ is positive semidefinite. Therefore, all principal submatrices are positive semidefinite and we have $l_{i i} \geq \tilde{l}_{i i}$. As a consequence of the substitution lemma, we know that $\sum_{j \in B: i \neq j} \tilde{\omega}_{i j}=\tilde{l}_{i i}$, and hence $\sum_{j \in B: i \neq j} \tilde{\omega}_{i j} \leq l_{i i}$. Since each of the $\tilde{\omega}_{i j}$ 's is positive, each summand has to be smaller than $l_{i i}$. By the same argument we can show that $\tilde{\omega}_{i j} \leq l_{j j}$ and the lemma follows.

The next lemma gives us a lower bound for $\tilde{\omega}_{s t}$ and tells us how we have to select the number $K$ such that $\tilde{\omega}_{s t}$ becomes the dominant stress.

Lemma 3. Let $\omega$ be an $x$-equilibrium stress obtained from the edge costs $c_{\{i, j\}}$ for $x_{1}, \ldots, x_{n}$ that is increased along an $x$-montone path from $v_{s}$ to $v_{t}$, by incrementing the edge costs for edges on the path by $K$. The substitution stresses and the matrix $L$ are obtained from $\omega$ as usual. Then

$$
\tilde{\omega}_{s t}>\frac{K-3 n^{2}\left(1+(k-2) \Delta_{x}\right)}{x_{t}-x_{s}},
$$

where $k$ denotes the number of boundary vertices and $\Delta_{x}$ the largest distance between two $x$-coordinates. Let $\alpha>0$ be a parameter that will be fixed later. For $K \geq 3 n^{2}\left(1+(\alpha+k-2) \Delta_{x}\right)$ we obtain for any $\tilde{\omega}_{i j}$ that is not $\tilde{\omega}_{s t}$

$$
\tilde{\omega}_{s t}>\alpha \tilde{\omega}_{i j}
$$

Proof. Before bounding $\tilde{\omega}_{s t}$ we show an upper bound for the other substitution stresses. Let $\tilde{\omega}_{i j}$ be such a stress. By Lemma 2, $\tilde{\omega}_{i j}$ is less than $l_{r r}$ $(r \in\{i, j\} \cap\{s, t\})$. Since $l_{r r}$ is a diagonal entry of the Laplace matrix it equals $\sum_{k:(r, k) \in E} \omega_{r k}$, which is a sum of at most $n-1$ summands. We can assume that the path $P_{s t}$ uses no boundary edge. In this case each summand in $\sum_{k:(r, k) \in E} \omega_{r k}$ is smaller than $3 n-3$ and we obtain

$$
\forall i j \neq s t \quad \tilde{\omega}_{i j}<\max _{r \in B \backslash\{s, t\}}\left\{l_{r r}\right\}<3 n^{2} .
$$

Let $F_{t}^{x}$ be the $x$-component of $\mathbf{F}_{t}$. We combine (9) and (4) and obtain as upper bound for $F_{t}^{x}$

$$
\tilde{\omega}_{s t}\left(x_{t}-x_{s}\right)+(k-2) 3 n^{2} \Delta_{x}>F_{t}^{x} .
$$

On the other hand we can express $F_{t}^{x}$ by (3). The cost of one edge $(k, t)$, with $x_{k}<x_{t}$, was increased by $K$. All other costs are in total less than $3 n^{2}$. Thus,

$$
F_{t}^{x}=\sum_{k:(k, t) \in E} \omega_{k t}\left(x_{t}-x_{k}\right)=\sum_{\substack{\left.k: x_{k}<x_{t} \\ k, t\right) \in E}} c_{\{t, k\}}-\sum_{\substack{k: x_{k}>x_{t} \\(k, t) \in E}} c_{\{t, k\}}>K-3 n^{2} .
$$


Combining the two bounds for $F_{t}^{x}$ leads to the bound for $\tilde{\omega}_{s t}$ stated in the lemma. Setting $K \geq 3 n^{2}\left(1+(\alpha+k-2) \Delta_{x}\right)$ yields

$$
\tilde{\omega}_{s t}>\frac{3 n^{2}(\alpha+k-2) \Delta_{x}-3 n^{2}(k-2) \Delta_{x}}{x_{t}-x_{s}}=\frac{3 n^{2} \alpha \Delta_{x}}{x_{t}-x_{s}} \geq 3 n^{2} \alpha>\alpha \tilde{\omega}_{i j} .
$$

As last part of this section we show that we can even enforce a set of substitution stresses to be dominant. Let $v_{t_{1}}, v_{t_{2}}$ be two vertices that are both nonincident to $v_{s}$ and which have the same $x$-coordinate. We can increment a given $x$-equilibrium stress by first increasing the edge $\operatorname{costs} c_{\{i, j\}}$ along an $x$-monotone path from $v_{s}$ to $v_{t_{1}}$ by $K$ and then do the same for an appropriate path from $v_{s}$ to $v_{t_{2}}$.

Lemma 4. Assume the same as in Lemma 3, but this time consider two paths: from $v_{s}$ to $v_{t_{1}}$ and from $v_{s}$ to $v_{t_{2}}$. Assume further that $x_{t_{1}}=x_{t_{2}}$. For $K \geq$ $3 n^{2}\left(1+(\alpha+k-2) \Delta_{x}\right)$ we have for any $\tilde{\omega}_{i j}$ with $\{i, j\} \not \subset\left\{s, t_{1}, t_{2}\right\}$

$$
\tilde{\omega}_{s t_{1}}>\alpha \tilde{\omega}_{i j} \text {, and } \tilde{\omega}_{s t_{2}}>\alpha \tilde{\omega}_{i j} \text {. }
$$

Proof. Lemma 3 relies on the fact that we can bound $\sum_{j \neq s} \tilde{\omega}_{j t}\left(x_{t}-x_{j}\right)$ because by (9) the $\tilde{\omega}^{\prime}$ s in this sum are small. We cannot use this bound for $\tilde{\omega}_{t_{1} t_{2}}\left(x_{t_{1}}-x_{t_{2}}\right)$ anymore, but this summand cancels anyway, since $x_{t_{1}}=x_{t_{2}}$. Following the steps of the proof of Lemma 3 with first choosing $t=t_{1}$ and then choosing $t=t_{2}$ shows the lemma.

\section{The Embedding Algorithm}

\subsection{The Algorithm Template}

In this section we present as main result of this paper

Theorem 2. Let $G$ be a 3-connected planar graph. $G$ admits an embedding as 3-polytope inside a $2(n-2) \times 1 \times 1$ box under the vertex resolution rule.

We prove Theorem 2 by introducing an algorithm that constructs the desired embedding. The number of vertices on the boundary face plays an important role. The smaller this number is, the simpler is it to construct an embedding. For this reason we choose as boundary face $f_{b}$ the smallest face of $G$. Due to Euler's formula this face has at most 5 vertices. Depending on the size of $f_{b}$ we obtain three versions of the algorithm which all follow the same basic pattern.

Our goal is to construct a planar embedding of $G$ that has a positive stress and whose $x$-coordinates are distinct integers. The corresponding lifting of such an embedding fulfills the vertex resolution rule independently of its $y$ and $z$ coordinates. Hence, we can scale in direction of the $y$ and $z$-axis without violating the vertex resolution rule. The basic procedure is summarized in Algorithm 5.1

Steps 1,4 , and 5 are mostly independent of the size of $f_{b}$ and will be discussed first. We start with some strictly convex plane embedding of $G$ - called 


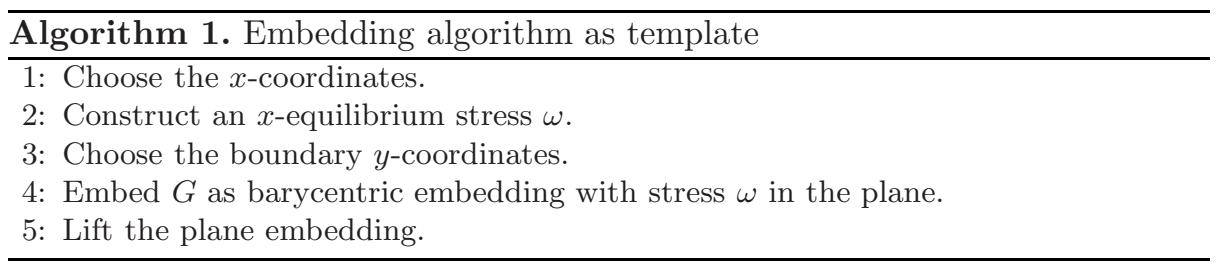

pre-embedding. Let $\hat{x}_{1}, \ldots, \hat{x}_{n}$ and $\hat{y}_{1}, \ldots, \hat{y}_{n}$ be the coordinates of the preembedding which we compute as barycentric embedding. We assume that in the pre-embedding all $x$-coordinates are different. If this is not the case we can perturb the stresses of the (pre)-embedding to achieve this. Since the pre-embedding is strictly convex [17] every edge lies on some $x$-monotone path. As done in Section 4 we fix for every interior edge $e$ such a path $P_{e}$. The $x$-coordinates of the pre-embedding induce a strict linear order on the vertices of $G$. We denote with $b_{i}$ the number of vertices with smaller $x$-coordinate compared to $v_{i}$ in the preembedding. The $x$-coordinates of the (final) embedding are defined as $x_{i}:=b_{i}$. Thus, no two vertices get the same $x$-coordinate and the largest $x$-coordinate is less than $n$. We observe that the paths $P_{e}$ remain $x$-monotone. Therefore, they can be used to define an appropriate $x$-equilibrium stress $\omega$. For technical reasons we might choose the same $x$-coordinate for some of the boundary vertices. In this case we check the vertex resolution rule for these vertices in the final embedding by hand. Step 4 and 5 can be realized as straight-forward implementations of the barycentric embedding and Maxwell's lifting. Notice that the value of the (extended) stress on the boundary edges is not needed to compute the lifting, because we can place an interior face in the $x y$-plane and then compute the lifting using only interior edges.

Steps 2 and 3 are the difficult parts of the algorithm. We have to choose the stress $\omega$ and the $y$-coordinates such that an extension of the stress to the boundary is possible. Moreover, the $y$-coordinates should give a convex boundary face. We continue with the three different cases and discuss step 2 and 3 of the algorithm template for each of them separately.

\subsection{Graphs with Triangular Face}

The case where $f_{b}$ is a triangle is the easiest case because we can extend every stress to the boundary for every location of the outer face (see Section 33). This case was already addressed by Chrobak, Goodrich and Tamassia 1]. The discussion in this section will prove the following statement:

Proposition 1. Let $G$ be a 3-connected planar graph and let $G$ contain a triangular face. $G$ admits an embedding as 3-polytope inside an $(n-1) \times 1 \times 1$ box under the vertex resolution rule.

Let us compute the pre-embedding with the boundary coordinates $x_{1}=0, x_{2}=$ 1 , and $x_{3}=0$. We use the $x$-monotone paths $P_{e}$ to compute a suitable $x$ equilibrium stress $\omega$ as discussed in Section 4 . Next we compute the barycentric 
embedding with boundary coordinates $\mathbf{p}_{1}=(0,0)^{T}, \mathbf{p}_{2}=(n-1,0)^{T}$, and $\mathbf{p}_{3}=$ $(0,1)^{T}$. As result we obtain interior $y$-coordinates in the interval $(0,1)$. Any two vertices have distance at least 1 , since their $x$-coordinates differ by at least 1 . (This is not true for $v_{1}$ and $v_{3}$, but their distance is $y_{3}-y_{1}=1$.)

\subsection{Graphs with Quadrilateral Face}

Let us assume now that $G$ contains a quadrilateral but no triangular face. In this case it is not always possible to extend an equilibrium stress $\omega$ to the boundary. The observations of Section 3 help us to overcome this difficulty. We use as boundary coordinates for the pre-embedding $\hat{x}_{1}=0, \hat{x}_{2}=1, \hat{x}_{3}=1$, and $\hat{x}_{4}=0$. The $x$-coordinates induced by the pre-embedding give $x_{2}=x_{3}=n-2$. We redefine the boundary $x$-coordinates by setting $x_{2}=n-2$ and $x_{3}=2(n-2)$. Notice that this preserves the $x$-monotonicity of the paths $P_{e}$, but makes it easier to extend the stress to the boundary as we will see in the following.

Let $\omega$ be the $x$-monotone stress for the obtained $x$-coordinates. We can express the influence of the stressed edges of $G$ on the boundary with help of the substitution stresses $\tilde{\omega}$. We modify $\omega$ with the technique described in Lemma 3 to assure that $\tilde{\omega}_{13}>\tilde{\omega}_{24}$. Let us now discuss how to extend the stress $\omega$ to the boundary. To solve the non-linear system given by (4) and (5) we fix some of the boundary $y$-coordinates to obtain a unique solution. In particular, we set $y_{1}=0, y_{2}=0$, and $y_{4}=1$. As final coordinate we obtain 1

$$
y_{3}=\frac{\tilde{\omega}_{24}}{2 \tilde{\omega}_{13}-\tilde{\omega}_{24}} .
$$

Since $\tilde{\omega}_{13}>\tilde{\omega}_{24}$, we can deduce that $0<y_{3}<1$. Hence, all $y$-coordinates are contained in $[0,1]$. Furthermore, we know that the only two vertices with the same $x$-coordinate, namely $v_{1}$ and $v_{4}$, have the distance $y_{4}-y_{1}=1$. Therefore the vertex resolution rule holds. If we scale the induced lifting such that the largest $z$-coordinate equals 1 we obtain:

Proposition 2. Let $G$ be a 3-connected planar graph and let $G$ contain a quadrilateral face. $G$ admits an embedding as 3-polytope inside a $2(n-2) \times 1 \times 1$ box under the vertex resolution rule.

\subsection{The General Case}

The most complicated case is the case where we have to use a pentagon as boundary face. However, the basic pattern how to construct the embedding remains the same. We choose as $x$-coordinates for the pre-embedding $\hat{x}_{1}=0, \hat{x}_{2}=$ $1, \hat{x}_{3}=1, \hat{x}_{4}=0$, and $\hat{x}_{5}=-\varepsilon$, for $\varepsilon>0$ small enough to guarantee that all interior vertices get a positive $x$-coordinate in the pre-embedding. We change the induced $x$-coordinates on the boundary without changing the monotonicity of the paths $P_{e}$. In particular, we set $x_{1}=0, x_{2}=n-2, x_{3}=n-2, x_{4}=0$, and

\footnotetext{
${ }^{1}$ The solution of the non-linear system was obtained by computer algebra software.
} 
$x_{5}=-(n-2)$. The stress $\omega$ is constructed such that the substitution stresses guarantee

$$
\tilde{\omega}_{25}>3 \tilde{\omega}_{13}, \tilde{\omega}_{25}>3 \tilde{\omega}_{14}, \tilde{\omega}_{25}>3 \tilde{\omega}_{24}, \tilde{\omega}_{35}>3 \tilde{\omega}_{13}, \tilde{\omega}_{35}>3 \tilde{\omega}_{14}, \tilde{\omega}_{35}>3 \tilde{\omega}_{24} .
$$

In other words the substitution stresses $\tilde{\omega}_{25}$ and $\tilde{\omega}_{35}$ dominate all other substitution stresses on interior edges by a factor 3 . Since $x_{3}=x_{2}$, we can construct a stress $\omega$ that induces a substitution stress that fulfills (10) by the observations of Lemma 4 .

Appropriate boundary $y$-coordinates can be obtained by solving the nonlinear system given by (4) and (5). As done in the previous case we fix some $y$-coordinates to obtain a unique solution. This time we set $y_{1}=-1, y_{4}=1$, and $y_{5}=0$. This yields for the two remaining $y$-coordinates

$$
\begin{aligned}
& y_{2}=-2-2 \frac{\tilde{\omega}_{24} \tilde{\omega}_{13}-\tilde{\omega}_{13}^{2}-\tilde{\omega}_{35} \tilde{\omega}_{14}-2 \tilde{\omega}_{13} \tilde{\omega}_{35}}{\tilde{\omega}_{24} \tilde{\omega}_{35}+\tilde{\omega}_{25} \tilde{\omega}_{13}+2 \tilde{\omega}_{25} \tilde{\omega}_{35}}, \\
& y_{3}=2+2 \frac{\tilde{\omega}_{24} \tilde{\omega}_{13}-\tilde{\omega}_{24}^{2}-\tilde{\omega}_{14} \tilde{\omega}_{25}-2 \tilde{\omega}_{24} \tilde{\omega}_{25}}{\tilde{\omega}_{24} \tilde{\omega}_{35}+\tilde{\omega}_{25} \tilde{\omega}_{13}+2 \tilde{\omega}_{25} \tilde{\omega}_{35}} .
\end{aligned}
$$

We have to check two things: $f_{b}$ has to be convex and $y_{3}-y_{2}$ should be large enough to guarantee the vertex resolution rule. First we show that $-2<y_{2}$ and $y_{3}<2$, which would imply that $f_{b}$ is convex if $y_{3}>y_{2}$. The inequalities $-2<y_{2}$ and $y_{3}<2$ hold, iff

$$
\begin{aligned}
& \tilde{\omega}_{24} \tilde{\omega}_{13}-\tilde{\omega}_{13}^{2}-\tilde{\omega}_{35} \tilde{\omega}_{14}-2 \tilde{\omega}_{35} \tilde{\omega}_{13}<0 \text { and } \\
& \tilde{\omega}_{24} \tilde{\omega}_{13}-\tilde{\omega}_{24}^{2}-\tilde{\omega}_{14} \tilde{\omega}_{25}-2 \tilde{\omega}_{25} \tilde{\omega}_{24}<0 .
\end{aligned}
$$

Both inequalities are true, because as a consequence of (10) the only positive summand $\tilde{\omega}_{24} \tilde{\omega}_{13}$ is smaller than $\tilde{\omega}_{35} \tilde{\omega}_{13}$ and smaller than $\tilde{\omega}_{25} \tilde{\omega}_{24}$. The difference $y_{3}-y_{2}$ equals

$$
4+2 \frac{2 \tilde{\omega}_{24} \tilde{\omega}_{13}-\tilde{\omega}_{13}^{2}-\tilde{\omega}_{24}^{2}-\tilde{\omega}_{35}\left(\tilde{\omega}_{14}+2 \tilde{\omega}_{13}\right)-\tilde{\omega}_{25}\left(\tilde{\omega}_{14}+2 \tilde{\omega}_{24}\right)}{\tilde{\omega}_{24} \tilde{\omega}_{35}+\tilde{\omega}_{25} \tilde{\omega}_{13}+2 \tilde{\omega}_{25} \tilde{\omega}_{35}} .
$$

Due to (10) we know that $\tilde{\omega}_{14}+2 \tilde{\omega}_{24}<\tilde{\omega}_{35}$ and $\tilde{\omega}_{14}+2 \tilde{\omega}_{13}<\tilde{\omega}_{25}$. Thus

$$
y_{3}-y_{2}>4+2 \frac{2 \tilde{\omega}_{24} \tilde{\omega}_{13}-\tilde{\omega}_{13}^{2}-\tilde{\omega}_{24}^{2}-2 \tilde{\omega}_{35} \tilde{\omega}_{25}}{\tilde{\omega}_{24} \tilde{\omega}_{35}+\tilde{\omega}_{25} \tilde{\omega}_{13}+2 \tilde{\omega}_{25} \tilde{\omega}_{35}}>2 .
$$

The estimation holds since (again due to (10) $) \tilde{\omega}_{24} \tilde{\omega}_{35}>\tilde{\omega}_{24}^{2}$ and $\tilde{\omega}_{25} \tilde{\omega}_{13}>\tilde{\omega}_{13}^{2}$, and hence the fraction is greater -1 .

We multiply all $y$-coordinates by $1 / 2$. This yields $y_{4}-y_{1}=1$ and $y_{3}-y_{2}>1$. The $z$-coordinates are scaled such that they lie between 0 and 1 . Clearly, the vertex resolution rule holds for the computed embedding.

We finish this section with some remarks on the running time of the embedding algorithm. As mentioned in [1] the $x$-monotone stress can be computed in linear time. The barycentric embedding (which we use twice, once for the preembedding and once for the intermediate plane embedding) can be computed 
by the linear system (2). Since the linear system is based on a planar structure, we can solve it with nested dissections (see 910]) based on the planar separator theorem. As a consequence a solution can be computed in $O(M(\sqrt{n}))$ time, where $M(n)$ is the upper bound for multiplying two $n \times n$ matrices. The current record for $M(n)$ is $O\left(n^{2.325}\right)$, which is due Coppersmith and Winograd [2]. The computation of the lifting can be done in linear time. In total we achieve a running time of $O\left(n^{1.1625}\right)$.

\section{Additional Properties of the Embedding}

\subsection{Induced Grid Embedding}

Besides the small embedding under the vertex resolution rule, the constructed embedding has several other nice properties which we discuss in this section. Since the computed $y$ and $z$-coordinates are expressed by a linear system of rational numbers, they are rational numbers as well. Thus, we can scale the final embedding to obtain a grid embedding.

Theorem 3. The embedding computed with Algorithm 5.1 can be scaled to integer coordinates such that

$$
\begin{aligned}
& 0 \leq x_{i} \leq 2(n-2) \\
& 0 \leq y_{i}, z_{i} \leq 2^{O\left(n^{2} \log n\right)}
\end{aligned}
$$

The proof of the theorem can be found in the full version of the paper. Compared to the grid embedding presented in [12], we were able to reduce the size of the $x$-coordinates (from $2 n \cdot 8.107^{n}$ to $2(n-2)$ ) at the expense of the $y$ and $z$ coordinates.

\subsection{Spread of the Embedding}

The spread of a point set is the quotient of the longest pairwise distance (the diameter) and the shortest pairwise distance. The smaller this ratio is, the more densely the point set is packed. A small spread implies that the points are "evenly distributed". We define as spread of an embedding of a polytope the spread of its points.

Theorem 4. The spread of a 3-polytope embedded by Algorithm 5.1 is smaller than $n$. There are infinitely many polytopes without an embedding with spread smaller than $(n-1) / \pi$.

The proof of the theorem can be found in the full version of the paper.

\section{References}

1. Chrobak, M., Goodrich, M.T., Tamassia, R.: Convex drawings of graphs in two and three dimensions (preliminary version). In: 12th Symposium on Computational Geometry, pp. 319-328 (1996) 
2. Coppersmith, D., Winograd, S.: Matrix multiplication via arithmetic progressions. J. Symb. Comput. 9(3), 251-280 (1990)

3. Crapo, H., Whiteley, W.: Plane self stresses and projected polyhedraI: The basic pattern. Structural Topology 20, 55-78 (1993)

4. Das, G., Goodrich, M.T.: On the complexity of optimization problems for 3-dimensional convex polyhedra and decision trees. Comput. Geom. Theory Appl. 8(3), 123-137 (1997)

5. de Fraysseix, H., Pach, J., Pollack, R.: How to draw a planar graph on a grid. Combinatorica 10(1), 41-51 (1990)

6. Eades, P., Garvan, P.: Drawing stressed planar graphs in three dimensions. In: Brandenburg, F.J. (ed.) GD 1995. LNCS, vol. 1027, pp. 212-223. Springer, Heidelberg (1996)

7. Gortler, S.J., Gotsman, C., Thurston, D.: Discrete one-forms on meshes and applications to 3d mesh parameterization. Computer Aided Geometric Design 23(2), 83-112 (2006)

8. Hopcroft, J.E., Kahn, P.J.: A paradigm for robust geometric algorithms. Algorithmica $7(4), 339-380(1992)$

9. Lipton, R.J., Rose, D., Tarjan, R.: Generalized nested dissection. SIAM J. Numer. Anal. 16(2), 346-358 (1979)

10. Lipton, R.J., Tarjan, R.E.: Applications of a planar separator theorem. SIAM J. Comput. 9(3), 615-627 (1980)

11. Maxwell, J.C.: On reciprocal figures and diagrams of forces. Phil. Mag. Ser. 27, 250-261 (1864)

12. Ribó Mor, A., Rote, G., Schulz, A.: Small grid embeddings of 3-polytopes (2009) (submitted for publication), http://arxiv.org/abs/0908.0488

13. Richter-Gebert, J.: Realization Spaces of Polytopes. Lecture Notes in Mathematics, vol. 1643. Springer, Heidelberg (1996)

14. Schnyder, W.: Embedding planar graphs on the grid. In: Proc. 1st ACM-SIAM Sympos. Discrete Algorithms, pp. 138-148 (1990)

15. Schramm, O.: Existence and uniqueness of packings with specified combinatorics. Israel J. Math. 73, 321-341 (1991)

16. Steinitz, E.: Encyclopädie der mathematischen Wissenschaften. In: Polyeder und Raumteilungen, pp. 1-139 (1922)

17. Tutte, W.T.: How to draw a graph. Proceedings London Mathematical Society $13(52), 743-768$ (1963)

18. Whiteley, W.: Motion and stresses of projected polyhedra. Structural Topology 7, 13-38 (1982)

19. Whitney, H.: A set of topological invariants for graphs. Amer. J. Math. 55, 235-321 (1933)

20. Zhang, F.: Matrix Theory. Springer, Heidelberg (1999) 